\title{
An agent-based variant of the standard hidden-action model
}

\author{
Stephan Leitner ${ }^{*}$ Friederike Wall ${ }^{* *}$ \\ * Department of Management Control and Strategic Management, \\ Alpen-Adria-Universität Klagenfurt, Universitätsstr. 65-67, 9020 \\ Klagenfurt, Austria (e-mail: stephan.leitner@aau.at). \\ ** Department of Management Control and Strategic Management, \\ Alpen-Adria-Universität Klagenfurt, Universitätsstr. 65-67, 9020 \\ Klagenfurt, Austria (e-mail: friederike.wall@aau.at).
}

Keywords: Simulation, numerical simulation, economics, learning, dynamic system, management.

\section{INTRODUCTION}

In the context of incomplete information and environmental uncertainty, normative agency theory focuses on finding the most efficient contract between the principal and the agent, that aligns the two parties' interests but also maximizes the principal's utility. Typical classes of problems investigated within the framework of agency theory are concerned with hidden-characteristics, hiddeninformation and hidden-action (e.g., Eisenhardt, 1989).

Normative agency models have a set of assumptions incorporated. These assumptions are mainly concerned with (the distribution of) information and the involved individuals' behaviour (e.g., Müller, 1995) and might be regarded as a virtue as they allow for deriving optimal contracts in closed-form modeling. At the same time, these assumptions might also be a fundamental weakness as they might limit the theory's predictive validity.

The positive agency literature calls for relaxing these assumptions to map real-world situations. There are some conceptual papers that make aware of the limitations of normative agency literature (e.g., Shapiro, 2005). In addition, there is some empirical research that shows that mechanisms derived from normative agency models fail to work in real-world situations (e.g., Cuevas-Rodriguez et al., 2012). This is where we particularly place our research: We aim at contributing to closing the gap between positive and normative agency literature by providing a systematic analysis of selected assumptions in the standard hidden-action model.

In this paper, we transfer the standard hidden-action model into an agent-based variant of the hidden-action problem. In order to do so, we employ the so-called agentization approach which allows us to relax selected assumption incorporated in the standard hidden-action model. In the current paper, the process of agentization particularly concentrates on assumptions regarding the information available for both the principal and the agent. While the standard model allows to find the optimal contract in one timestep, the agent-based model variant requires the involved parties to search for the optimal contract over time. In addition to relaxed assumptions, the proposed agent-based model variant endows the principal and the agent with learning capabilities and a memory in which the learnings can be stored. The principal is additionally endowed with an exploration propensity which drives the selection of the strategy employed to search for 'better' contracts.

\section{THE MODEL IN A NUTSHELL}

We refer to the following model as standard hidden-action model: The principal offers the agent a contract (inter alia consisting of a task to be executed and a compensation scheme). In case the agent accepts the contract, she autonomously selects an effort level to execute the task. Together with an exogenous factor the selected effort level defines the outcome, which is observable by both the principal and the agent. The principal can neither observe the selected effort level, nor does he have information on the exogenous factor. Thus, the agent's compensation can only be based on the outcome. The standard hiddenaction model gives information on how the contract should be designed so that the principal's utility maximizes (cf. Lambert, 2001).

The principal's utility function can be formalized by

$$
U_{P}(\overbrace{\underbrace{X(a, \rho, \theta)}_{x=a \cdot \rho+\theta}}^{\text {production function }}, \overbrace{\underbrace{S\left(c_{f}, x, p\right)}_{s=c_{f}+x \cdot p}}^{\text {compensation function }}),
$$

while the agent's utility function can be formalized by

$$
U_{A}(s, a)=\overbrace{V(s)}^{\begin{array}{c}
\text { utility from } \\
\text { compensation }
\end{array}}-\overbrace{G(a)}^{\begin{array}{c}
\text { disutility from } \\
\text { exerting effort }
\end{array}},
$$

where $a \in A$ represents the set of possible actions the agent can select from to carry out the delegated task, $\rho$ stands for the agent's productivity, $\theta \in \Theta$ indicates the exogenous factor, $c_{f}$ stands for the agent's fixed compensation component, and $p$ indicates a premium level. For further elaborations, the agent's reservation utility is indicated by $\underline{U}$. 
The principal is modeled to seek to maximize his expected utility (see Eq. 3) subject to the participation (see Eq. 4) and incentive compatibility constraint (see Eq. 5): ${ }^{1}$

$$
\begin{array}{ll}
\max & \mathbb{E}\left(U_{P}(x, s)\right) \\
\text { s.t. } & \mathbb{E}\left(U_{A}(s, a)\right) \geq \underline{U} \\
& \int V(s) f_{a}(x \mid a) d x-G(a)^{\prime}=0
\end{array}
$$

The agent is also modeled to seek to maximize the expected utility,

$$
\max \mathbb{E}\left(U_{A}(s, a)\right)
$$

Agency theory allows the principal to solve the optimization problem (i.e., to find the 'optimal' premium level, $p$, cf. Eq. 3-5) within one timestep, which (sometimes implicitly) includes very specific assumptions about the availability of information as well as about the involved parties capabilities. It is, e.g., assumed that the principal has full information about the agent's characteristics $\left(U_{A}, \underline{U}, \rho\right)$, is able to observe the outcome $(x)$, knows the entire set of actions $(A)$ the agent can select from to carry out the delegated task, and has information about the distribution of exogenous factors $(\Theta)$. The agent is assumed to have information on the distribution on exogenous factors $(\Theta)$, and the outcome $(x)$. In addition, the agent is modeled to have private information on the selected action $(a)$ and the realized exogenous factor $(\theta){ }^{2}$

Using the so-called agentization approach, the standard model is transferred into an agent-based model variant. ${ }^{3}$ The agent-based model variant represents a multi-period version of the standard hidden-action model. A special feature of the agent-based model variant is that the principal and the agent are no longer able to find the optimal solution in one time-step but rather have to search for the optimal solution over time. During the process of agentization, particular focus is put on assumptions regarding the principal's and the agent's information about the distribution of exogenous factors and the principal's information about the set of actions. In particular, regarding the information about the action space and the distribution of exogenous factors, the following (presumably more realistic) assumptions are included in the agent-based model variant:

\section{Assumptions regarding the principal's information}

- Principal has limited information about the set of actions

- Principal is endowed with a mental horizon which defines the fraction of $A$ that can be overseen

- Principal has no information about the distribution of exogenous factors

- Principal is endowed with the capability to learn about the distribution of exogenous factors over time and with a memory in which learnings are stored

\footnotetext{
1 For more details on the standard model and the included constraints the reader might consult Lambert (2001).

2 A detailed discussion of these assumptions is provided in Müller (1995).

3 For details on the agentization approach the reader is referred to Leitner and Behrens (2014) and Guerrero and Axtell (2011).
}

- Principal is endowed with an exploration propensity which drives the strategy employed to search for 'the optimal solution' over time

Assumptions regarding the agent's information:

- Agent has no information about the distribution of exogenous factors

- Agent is endowed with the capability to learn about the distribution of exogenous factors over time and with a memory in which learnings are stored

All other assumptions remain unaffected by the agentization approach and are carried over from the standard model to the agent-based model variant.

\section{SELECTED RESULTS}

We find that the impact of the exogenous factor on the task's outcome significantly affects the efficiency of the contract offered to the agent, i.e., performance decreases as the impact of the exogenous factor on the task's outcome increases. Our results also indicate that the principal's exploration propensity does not significantly affect the efficiency of the derived contract if the impact of the exogenous factor is kept stable. We do, however, observe significant differences (caused by the principal's exploration propensity) across scenarios in which the impact of the exogenous factor is varied. We find that, in our model, a lower level of exploration propensity is particularly beneficial when the environment has a strong impact on the task's outcome. Moreover, we aim at identifying additional factors that drive our results (like, e.g., the principal's mental horizon). Based on our results we aim at characterizing critical factors and their interrelations in the context of hidden-action problem and at providing decision support on how to optimally shape delegation relationships.

\section{REFERENCES}

Cuevas-Rodriguez, G., Gomez-Mejia, L.R., and Wiseman, R.M. (2012). Has agency theory run its course?: Making the theory more flexible to inform the management of reward systems. Corporate Governance: An International Review, 20(6), 526-546.

Eisenhardt, K.M. (1989). Agency theory: An assessment and review. Academy of Management Review, 14(1), $57-74$.

Guerrero, O.A. and Axtell, R. (2011). Using agentization for exploring firm and labor dynamics: A methodological tool for theory exploration and validation. In S. Osinga, G.J. Hofstede, and T. Verwaart (eds.), Emergent Results of Artificial Economics, volume 652 of Lecture Notes on Economics and Mathematical Systems, 139150. Springer, Berlin, Heidelberg.

Lambert, R.A. (2001). Contracting theory and accounting. Journal of Accounting and Economics, 32(1-3), 3-87.

Leitner, S. and Behrens, D.A. (2014). On the efficiency of hurdle rate-based coordination mechanisms. Mathematical and Computer Modelling of Dynamical Systems, $1-19$.

Müller, C. (1995). Agency-Theorie und Informationsgehalt. Die Betriebswirtschaft, 1995(1), 61-76.

Shapiro, S.P. (2005). Agency theory. Annual Review of Sociology, 31, 263-284. 